\title{
The Analysis of Online Medical Community Knowledge Sharing
}

\author{
Fan Xiaoniu \\ ${ }^{1}$ Xidian University, School of Economic and Management, Xi'an City, Xifeng Road, Xinglong266, China
}

\begin{abstract}
Based on evolutionary game theory, an game model is establishment that is about doctor and patient knowledge sharing behavior on online medical community. Making analysis of evolutionary path, evolutionary equilibrium, influencing factors and doing simulation with Matlab. The study found that medical is different with other field and that privacy leakage risk and reputation gains factors are the main factors influencing the evolution results and trust can offset part of the cost. Finally, give some advices combined with reality.
\end{abstract}

Keywords: Online medical community, Evolutionary game theory, knowledge sharing, hospital, patient

\section{Introduction}

The developing of the Internet is changing traditional medical treatment, to some degree, medical Internet mode has decreased the press and unbalance of medical resource. In our country many online medical sites such as haodf, dxy, chunyuyisheng, xywy offer Doctor-patient interaction capabilities to patients [1]. It makes doctors can know their patients' conditions on the Internet instead of unnecessary visits. While, comparing with traditional face-to-face treatment it results in a lower credibility. The technical restriction also keeps it away from observation, listening, interrogation, and pulse-taking which are the four traditional methods of diagnosis. At present, many of the online medical treatments do not get desired effect. Netizens think that it has a low probability which doctors can make reply in time. That's why they aren't satisfied with doctors. The disorder of the online medical treatments also leads to the query about the doctor's reliability. Above all, we will search how to raise the efficiency of the Online Medical Community Knowledge Sharing and solve these unsatisfied problems from knowledge sharing.

Scholars from domestic and overseas have tried to cognize and research the online medical treatment from different ways. The online medical community applies knowledge sharing and cooperating platform from the standpoint of knowledge management [2]. Users can not only share information and communicate with each other but also can influence each other [3]. What's more, there are lots of scholars searching for the emotion and behavior of the online medical community $[4,5,6]$. In our country XiaoxiaoLiu has found serious information asymmetry which exists in the online medical community and doctors' endeavor and prestige has a significant impact on the quantity of consultation [7]. Yang thinks in the online medical community, we can get the emotional support and health self-recovery [8]. Greene researches it by the qualitative analytical methods [9]. Well, Sillence compares with the free online medical consultation services in different countries and makes corresponding evaluations [10]. Cohen believes this way can improve the quality of care [11]
We research the online mutual problem from knowledge sharing. The online consult participators are both doctors and patients. The effectiveness is involved with both of their behaviors. And we must pay attention that their rationality is limited. The online medical community is an example of knowledge sharing. It's an ovonic process. Analyzing evolution path and evolution balance by creating a knowledge sharing evolution game model can help us get the factors which influence the doctor and patients' mutual positivity. We will use Matlab to simulate the evolution model.

\section{Create The Evolutionary Game Model}

\section{(1)Participants}

We choose the people who share their knowledge to each other by using the online medical community to consult problems. Everyone has two choices which are knowledge sharing and no knowledge sharing"

\section{(2)Research purpose is knowledge sharing.}

Everyone has two strategies, but we aim at how to use them to improve knowledge sharing.

\section{(3)The gain matrix}

$K_{i}$ is expressed as the amount of knowledge sharing, such as

$K_{1}$ is the amount of knowledge that patients' states of their diseases, $K_{2}$ is the amount of knowledge that doctors' treat diseases.

$C_{i}$ is expressed as the cost of knowledge sharing . ${ }^{C_{1}}$ is the patient's cost, time and knowledge encoding in the online consulting. $C_{2}$ is the doctor's time , knowledge encoding and requiring efforts, as knowledge accumulation.

$F$ is expressed as risks of patients' privacy blabbing. It's direct ratio the quality of patients'knowledge sharing . the 


\section{International Journal of Science and Research (IJSR) \\ ISSN (Online): 2319-7064 \\ Index Copernicus Value (2013): 6.14 | Impact Factor (2015): 6.391}

patient's knowledge sharing risk is $K_{1} F$; because medical treatment knowledge has high specialty, the doctor doesn't lost his specialty.

$\tau_{i}$ is expressed as the trust level. The higher trust level means the bigger cooperation profit. $\mu_{i}$ is expressed as the knowledge cooperation factor. In the communication both the doctor and the patient will have a deeper understanding of the process of the disease. Their knowledge cooperation factors are expressed as $\tau_{1} \mu_{1} K_{2}$ and $\tau_{2} \mu_{2} K_{1}$.

$\beta_{i}$ is expressed as knowledge absorptive capacity. In the online communication words and pictures are used to communicate. Because knowledge has the tacitness and everyone's capacity of learning is different, district earning of the doctor and the patient got from the knowledge sharing are marked as $\beta_{1} K_{2}$ and $\beta_{2} K_{1}$.

$\mathrm{P}$ is expressed as the patient's effectiveness, his or her own physical condition after taking doctor's advice. $\mathrm{E}$ is expressed as expected revenue. When the patient chooses paid consulting, if it is necessary he will see a doctor in a hospital. D is expressed as public praise. A good public praise will take the revenue $\mathrm{D}$ to the doctor, while a bad public praise will take the loss $-n D(\mathrm{n}>=1)$ to him.

We suppose the probability that the patient chooses knowledge sharing is $\mathrm{x}$ ( $0 \leq X \leq 1$ ), the probability that the doctor chooses knowledge sharing is y $(0 \leq Y \leq 1)$. According to the hypothesis above, we can get the income matrix of both sides, as shown in table 1 .

Table 1: Knowledge sharing income matrix of the patient and the doctor

\begin{tabular}{|c|c|c|c|}
\hline \multicolumn{2}{|c|}{} & \multicolumn{2}{c|}{ doctor } \\
\cline { 3 - 4 } \multicolumn{2}{|c|}{} & $\begin{array}{c}\text { Knowledge } \\
\text { sharing (y) }\end{array}$ & $\begin{array}{r}\text { no knowledge } \\
\text { sharing (1-y) }\end{array}$ \\
\hline \multirow{4}{*}{ patien } & Knowledge & $\begin{array}{c}\tau_{1} \mu_{1} K_{2}+\beta_{1} K_{2} \\
-C_{1}+P-K_{1} F\end{array}$ & $-C_{1}$, \\
$t$ & sharing (x) & $\tau_{2} \mu_{2} K_{1}+\beta_{2} K_{1}$ \\
& $-C_{2}+E_{2}+D$ & $\beta_{2} K_{1}-n D$ \\
& & $\beta_{1} K_{2},-C_{2}$ & 0,0 \\
\cline { 2 - 4 } & no knowledge & & \\
& sharing(1-x) & & \\
\hline
\end{tabular}

As time goes on, the probabilities $\mathrm{X}$ and $\mathrm{Y}$ that the doctor and the patient choose knowledge sharing will remain stable finally. So we can build the evolutionary game model by solving the replicated dynamic equation.

The effectiveness when patient adopt to knowledge sharing strategy is

$U_{11}=y\left(\tau_{1} \mu_{1} K_{2}+\beta_{1} K_{2}-C_{1}+P-K_{1} F\right)+(1-y)\left(-C_{1}\right)$

The effectiveness when patient adopt to no knowledge sharing strategy is

$U_{12}=y\left(\beta_{1} K_{2}\right)$

The average effectiveness of patient is

$\overline{\mathrm{U}}_{1}=x \mathrm{U}_{11}+(1-x) \mathrm{U}_{12}(3)$

The patient's replicated dynamic equation

$F(x)=\frac{d x}{d t}=x\left(U_{11}-\bar{U}_{1}\right)=x(1-x)\left(-C_{1}+y\left(\tau_{1} \mu_{1} K_{2}+P-K_{1} F\right)\right.$

According to evolutionary stable strategy of the replicated dynamic equation, that it is ESS, we will keep the stable point $x^{*}$ contents $\frac{d x^{*}}{d t}=0$ and the derivative of $\frac{d x}{d t}$ is less than zero at the point $x^{*}$.

In a similar way, we will get the replicated dynamic

equation of the doctor. $F(y)=\frac{d y}{d t}=y\left(U_{21}-\bar{U}_{2}\right)$

$$
=y(1-y)\left(-C_{2}+x\left(E_{2}+\tau_{2} \mu_{2} K_{1}+(\mathrm{n}+1) \mathrm{D}\right)\right)
$$

According to evolutionary stable strategy of the replicated dynamic equation, that it is ESS, we will keep the stable point $y^{*}$ contents $\frac{d y^{*}}{d t}=0$ and the derivative of $\frac{d y}{d t}$ is less than zero at the point $y^{*}$.

By keeping $F(x)=\frac{d x}{d t}=0, F(y)=\frac{d y}{d t}=0$, we can get five stable points of the system, as $\mathrm{O}(0,0) \mathrm{A}(0,1) \mathrm{B}(1.1) \mathrm{C}(1,0) \mathrm{D}\left(x^{*}\right.$, $\left.y^{*}\right)$

$x^{*}=\frac{C_{2}}{E_{2}+\tau_{2} \mu_{2} K_{1}+(\mathrm{n}+1) \mathrm{D}} y^{*}=\frac{C_{1}}{\tau_{1} \mu_{1} K_{2}+P-K_{1} F}$

The system has five part stable points, as O A B C D, their stability can be got by analysis the stability of the system's jacobian matrix. The jacobian matrix is 


\section{International Journal of Science and Research (IJSR) \\ ISSN (Online): 2319-7064}

Index Copernicus Value (2013): 6.14 | Impact Factor (2015): 6.391

$J=\left(\begin{array}{cc}\frac{\partial F(x)}{\partial x} & \frac{\partial F(x)}{\partial y} \\ \frac{\partial F(y)}{\partial x} & \frac{\partial F(y)}{\partial y}\end{array}\right)=$

$\left((1-2 x)\left(-C_{1}+y\left(\tau_{1} \mu_{1} K_{2}+P-K_{1} F\right)\right) \quad x(1-\mathrm{x})\left(\tau_{1} \mu_{1} K_{2}+P-K_{1} F\right)\right.$

$y(1-\mathrm{y})\left(E_{2}+\tau_{2} \mu_{2} K_{1}+(\mathrm{n}+1) \mathrm{D}\right) \quad(1-2 \mathrm{y})\left(-C_{2}+x\left(E_{2}+\tau_{2} \mu_{2} K_{1}+(\mathrm{n}+1) \mathrm{D}\right)\right)(6)$

The determinant value of $J$ is marked as $\operatorname{det} J$, trace is marked as $\operatorname{tr} J$, when $\operatorname{det} J>0$ and $\operatorname{tr} J<0$, the stable point is ESS. While det $J>0$ and $\operatorname{tr} J>0$, the point isn't stable. When $\operatorname{det} J<0$, it is the saddle point.

\section{Model Analysis}

The replication dynamic analysis of the patient's strategy : on the basis of equation (1), when $x=0,1$ ory $=y^{*}, \frac{d x}{d t}=0$ is expressed that the proportion if the patient choose knowledge sharing is stable. When $y>y^{*}, x=1$ is the ESS equilibrium point; when $y<y^{*}, x=0$ is the ESS equilibrium point. When $y=y^{*}$,all of $\mathrm{x}$ are equilibrium points.

The phase image in picture one shows the dynamic tendency and stability of point $\mathrm{x}$. In the same way, we can get the result that according to equation (2), when $x>x^{*}$, $y=1$ is the ESS equilibrium point; when $x<x^{*}, y=0$ is the ESS equilibrium point; when $x=x^{*}$, all of point $y$ are stable. The phase image in picture two shows the dynamic tendency and stability of point $y$.
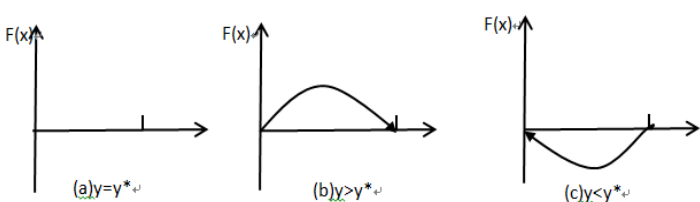

Figure 1:The copying dynamic phase diagram of patient
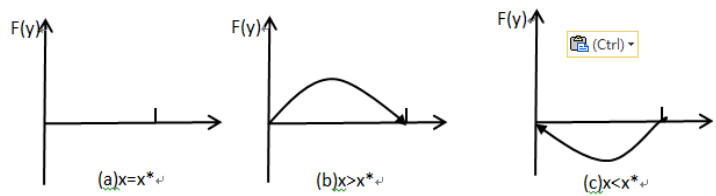

Figure 2:The copying dynamic phase diagram of doctor

Now we are going to analyze the condition when the revenue isn't equal to the cost. $1.0<x^{*}<1,0<y^{*}<1$ $\mathrm{C}_{1}+K_{1} F<\tau_{1} \mu_{1} K_{2}+P, \mathrm{C}_{2}<E_{2}+\tau_{2} \mu_{2} K_{1}+(\mathrm{n}+1) \mathrm{D} \quad$ Its reality meaning is that the revenue is bigger than the cost. The Local stability analysis result is shown in table 2.

Table2: when $\mathrm{C}_{1}+K_{1} F<\tau_{1} \mu_{1} K_{2}+P, \mathrm{C}_{2}<E_{2}+\tau_{2} \mu_{2} K_{1}+(\mathrm{n}+1) \mathrm{D}$ the analyzing result of system local stability

\begin{tabular}{|c|c|c|c|c|c|}
\hline $\begin{array}{l}\text { equilibrium } \\
\text { point }\end{array}$ & $\operatorname{det} J$ & symbol & trace of $J$ & symbol & local stability \\
\hline $\mathrm{O}(0,0)$ & $C_{1}{ }^{*} C_{2}$ & + & $-C_{1}-C_{2}$ & - & ESS \\
\hline $\mathrm{A}(0,1)$ & $\left(-C_{1}+\tau_{1} \mu_{1} K_{2}+P-K_{1} F\right) * C_{2}$ & + & $\left(-C_{1}+\tau_{1} \mu_{1} K_{2}+P-K_{1} F\right)+C_{2}$ & + & Instability point \\
\hline $\mathrm{B}(1,1)$ & $\begin{array}{l}\left(-C_{1}+\tau_{1} \mu_{1} K_{2}+P-K_{1} F\right)^{*} \\
\left(-C_{2}+E_{2}+\tau_{2} \mu_{2} K_{1}+(\mathrm{n}+1) \mathrm{D}\right)\end{array}$ & + & $\begin{array}{l}-\left(-C_{1}+\tau_{1} \mu_{1} K_{2}+P-K_{1} F\right) \\
-\left(-C_{2}+E_{2}+\tau_{2} \mu_{2} K_{1}+(\mathrm{n}+1) \mathrm{D}\right)\end{array}$ & - & ESS \\
\hline $\mathrm{C}(1,0)$ & $C_{1}^{*}\left(-C_{2}+E_{2}+\tau_{2} \mu_{2} K_{1}+(\mathrm{n}+1) \mathrm{D}\right)$ & + & $C_{1}^{*}\left(-C_{2}+E_{2}+\tau_{2} \mu_{2} K_{1}+(\mathrm{n}+1) \mathrm{D}\right)$ & + & Instability point \\
\hline $\mathrm{D}\left(\mathrm{x}^{*}, \mathrm{y}^{*}\right)$ & $U$ & - & 0 & 0 & saddle point \\
\hline
\end{tabular}

$U=-\frac{C_{2} C_{1}\left(E_{2}+\tau_{2} \mu_{2} K_{1}+(\mathrm{n}+1) \mathrm{D}-C_{2}\right)\left(\tau_{1} \mu_{1} K_{2}+P-K_{1} F-C_{1}\right)}{\left(E_{2}+\tau_{2} \mu_{2} K_{1}+(\mathrm{n}+1) \mathrm{D}\right) *\left(\tau_{1} \mu_{1} K_{2}+P-K_{1} F\right)}$

In the evolution system, the strategy of locally asymptotic stability becomes evolutionary equilibrium. For two users the evolution game of two strategies, evolutionary equilibrium is the same with ESS. When $0<x^{*}<1,0<y^{*}<1$, the system has five part equilibrium points. We analyzetheir part stability in table 2 . There are the meanings of each point in table 1. 


\section{International Journal of Science and Research (IJSR) \\ ISSN (Online): 2319-7064}

Index Copernicus Value (2013): 6.14 | Impact Factor (2015): 6.391

Table 3:The meaning of knowledge sharing system equilibrium points

\begin{tabular}{|c|c|c|}
\hline equilibrium point & result & meaning \\
\hline $\mathrm{O}(0,0)$ & ESS & There is no knowledge sharing between doctor and patient \\
\hline $\mathrm{A}(0,1)$ & instability point & doctor is knowledge sharing but patient is not knowledge sharing \\
\hline $\mathrm{B}(1,1)$ & ESS & here is knowledge sharing between doctor and patient \\
\hline $\mathrm{C}(1,0)$ & instability point & patientis knowledge sharing but doctor is not knowledge sharing \\
\hline $\mathrm{D}\left(\mathrm{x}^{*}, \mathrm{y}^{*}\right)$ & saddle point & the current state's evolution point of knowledge sharing \\
\hline
\end{tabular}

Using the phase path sketch map of the system shows the evolutionary process of knowledge sharing, as shown in table 1(1). When the beginning state is in quadrangle ADCB, the system converges to evolutionary stable strategy $\mathrm{B}(1,1)$. It means both of the users share their knowledge to each other. The bigger the area of ADCB is, the bigger the probability of the system converges to point $\mathrm{B}(1,1)$ is. So we can analyze the factor of the ADCB's area, and then we will get the influence factor of the system evolution path. The area of ADCB is:

$$
\begin{aligned}
& S_{A D B C}=\frac{1}{2}\left(2-x^{*}-y^{*}\right) \\
& =\frac{1}{2}\left(2-\frac{C_{2}}{E_{2}+\tau_{2} \mu_{2} K_{1}+(\mathrm{n}+1) \mathrm{D}}-\frac{C_{1}}{\tau_{1} \mu_{1} K_{2}+P-K_{1} F}\right)
\end{aligned}
$$

On the condition above, Long-term equilibrium result of the system evolution probably is that both sides share knowledge to each other or not share to each other. The evolution balanced state is related to the beginning state of the game and the critical point $\mathrm{D}\left(\mathrm{x}^{*}, \mathrm{y}^{*}\right)$. Now we will research how the change of several parameters influent the trend of system evolution.

(1) the costs of knowledge sharing $C_{1}$ and $C_{2}$ $\frac{\partial S_{A B C D}}{\partial C_{1}}=-\frac{1}{2 *\left(\tau_{1} \mu_{1} K_{2}+P-K_{1} F\right)}<0$

$\frac{\partial S_{A B C D}}{\partial C_{2}}=-\frac{1}{2 *\left(E_{2}+\tau_{2} \mu_{2} K_{1}+(n+1) D\right)}<0 \quad S_{A B C D}$ is the monotone decreasing function of $C_{1}$ and $C_{2}$. With the decrease of knowledge sharing cost, the area of $S_{A B C D}$ and the probability of knowledge sharing is bigger. At last, the system converges to the point $\mathrm{B}(1,1)$.

(2) $S_{A B C D}$ is the monotone increasing function of knowledge sharing quality, trust coefficient and synergy coefficient. With the parameters' values are increased, the area of $S_{A B C D}$ and the probability of knowledge sharing is bigger. At last, the system converges to the point $\mathrm{B}(1,1)$.

(3) The risk of knowledge sharing is shown as F. $\frac{\partial S_{A B C D}}{\partial F}=\frac{-C_{1} K_{1}}{2 *\left(\tau_{1} \mu_{1} K_{2}+P-K_{1} F\right)^{2}}<0 \quad S_{A B C D}$ is the monotone decreasing function of $\mathrm{F}$. With the risk of knowledge sharing is decreased, the area of $S_{A B C D}$ and the probability of knowledge sharing is bigger. At last, the system converges to the point $\mathrm{B}(1,1)$.

(4)The doctor reputation gains are shown as D. $\frac{\partial S_{A B C D}}{\partial K_{1}}=\frac{(\mathrm{n}+1) C_{2}}{2 *\left(E_{2}+\tau_{2} \mu_{2} K_{1}+(\mathrm{n}+1) \mathrm{D}\right)^{2}}>0$ $S_{A B C D}$ is the monotone increasing function of $\mathrm{D}$. With the qualities of their knowledge sharing are increased, the area of $S_{A B C D}$ and the probability of knowledge sharing is bigger. At last, the system converges to the point $\mathrm{B}(1,1)$.

2.

$$
0<x^{*}<1, y^{*}>1
$$

$\mathrm{C}_{1}+K_{1} F>\tau_{1} \mu_{1} K_{2}+P, \mathrm{C}_{2}<E_{2}+\tau_{2} \mu_{2} K_{1}+(\mathrm{n}+1) \mathrm{D}_{\text {their real }}$ meanings are the gains of the doctor is bigger than his cost because all of the patient's cost is more than his sharing gains. At present, $\mathrm{O}(0,0)$ is the equilibrium point. That is to say, the evolution result is both sides don't share knowledge. Local stability analysis results of the system are shown as table 4. The knowledge sharing dynamic evolution process is shown as figure $1(2)$. 


\section{International Journal of Science and Research (IJSR)}

ISSN (Online): 2319-7064

Index Copernicus Value (2013): 6.14 | Impact Factor (2015): 6.391

Table4: when $\mathrm{C}_{1}+K_{1} F>\tau_{1} \mu_{1} K_{2}+P, \mathrm{C}_{2}<E_{2}+\tau_{2} \mu_{2} K_{1}+(\mathrm{n}+1) \mathrm{D}$ the analyzing result of system local stability

\begin{tabular}{|c|c|c|c|c|c|}
\hline $\begin{array}{l}\text { equilibriu } \\
\text { m point }\end{array}$ & $\operatorname{det} J$ & symbol & trace of $J$ & symbol & local stability \\
\hline $\mathrm{O}(0,0)$ & $C_{1} * C_{2}$ & + & $-C_{1}-C_{2}$ & - & ESS \\
\hline $\mathrm{A}(0,1)$ & $\left(-C_{1}+\tau_{1} \mu_{1} K_{2}+P-K_{1} F\right)^{*} \mathrm{C}_{2}$ & - & $\left(-C_{1}+\tau_{1} \mu_{1} K_{2}+P-K_{1} F\right)+C_{2}$ & $\begin{array}{l}\text { unkno } \\
\text { wn }\end{array}$ & saddle point \\
\hline $\mathrm{B}(1,1)$ & $\begin{array}{l}\left(-C_{1}+\tau_{1} \mu_{1} K_{2}+P-K_{1} F\right)^{*} \\
\left(-C_{2}+E_{2}+\tau_{2} \mu_{2} K_{1}+(\mathrm{n}+1) \mathrm{D}\right)\end{array}$ & - & $\begin{array}{l}-\left(-C_{1}+\tau_{1} \mu_{1} K_{2}+P-K_{1} F\right) \\
-\left(-C_{2}+E_{2}+\tau_{2} \mu_{2} K_{1}+(\mathrm{n}+1) \mathrm{D}\right)\end{array}$ & $\begin{array}{l}\text { unkno } \\
\text { wn }\end{array}$ & saddle point \\
\hline$C(1,0)$ & $C_{1}^{*}\left(-C_{2}+E_{2}+\tau_{2} \mu_{2} K_{1}+(\mathrm{n}+1) \mathrm{D}\right)$ & + & $C_{1} *\left(-C_{2}+E_{2}+\tau_{2} \mu_{2} K_{1}+(\mathrm{n}+1) \mathrm{D}\right)$ & + & $\begin{array}{l}\text { Instability } \\
\text { point }\end{array}$ \\
\hline
\end{tabular}

On this condition, patients can build relationship with doctors actively and improve trust ranks. What's more, they can also offset part of the cost. Inequality conversion is shown as condition 1 .

3. $x^{*}>1,0<y^{*}<1$

$\mathrm{C}_{1}+K_{1} F<\tau_{1} \mu_{1} K_{2}+P, \mathrm{C}_{2}>E_{2}+\tau_{2} \mu_{2} K_{1}+(\mathrm{n}+1) \mathrm{D}$

their realmeanings are the gains of the patient is bigger than his cost. Doctor's costs are more than his sharing gains. At present, $\mathrm{O}(0,0)$ is the equilibrium point. That is to say, the evolution result is both sides don't share knowledge. Local stability analysis results of the system are shown as table 5 . The knowledge sharing dynamic evolution process is shown as figure $1(3)$.

Table5: When $\mathrm{C}_{1}+K_{1} F>\tau_{1} \mu_{1} K_{2}+P, \mathrm{C}_{2}<E_{2}+\tau_{2} \mu_{2} K_{1}+(\mathrm{n}+1) \mathrm{D}$ the analyzing result of system local stability

\begin{tabular}{|c|c|c|c|c|c|}
\hline $\begin{array}{l}\text { equilibriu } \\
\text { m point }\end{array}$ & $\operatorname{det} J$ & symbol & trace of $J$ & symbol & local stability \\
\hline $\mathrm{O}(0,0)$ & $C_{1} * C_{2}$ & + & $-C_{1}-C_{2}$ & - & ESS \\
\hline $\mathrm{A}(0,1)$ & $\left(-C_{1}+\tau_{1} \mu_{1} K_{2}+P-K_{1} F\right)^{*} C_{2}$ & + & $\left(-C_{1}+\tau_{1} \mu_{1} K_{2}+P-K_{1} F\right)+C_{2}$ & + & $\begin{array}{c}\text { Instability } \\
\text { point }\end{array}$ \\
\hline $\mathrm{B}(1,1)$ & $\begin{array}{l}\left(-C_{1}+\tau_{1} \mu_{1} K_{2}+P-K_{1} F\right)^{*} \\
\left(-C_{2}+E_{2}+\tau_{2} \mu_{2} K_{1}+(\mathrm{n}+1) \mathrm{D}\right)\end{array}$ & - & $\begin{array}{l}-\left(-C_{1}+\tau_{1} \mu_{1} K_{2}+P-K_{1} F\right) \\
-\left(-C_{2}+E_{2}+\tau_{2} \mu_{2} K_{1}+(\mathrm{n}+1) \mathrm{D}\right)\end{array}$ & unknown & Saddle point \\
\hline$C(1,0)$ & $C_{1} *\left(-C_{2}+E_{2}+\tau_{2} \mu_{2} K_{1}+(\mathrm{n}+1) \mathrm{D}\right)$ & - & $C_{1} *\left(-C_{2}+E_{2}+\tau_{2} \mu_{2} K_{1}+(\mathrm{n}+1) \mathrm{D}\right)$ & unknown & Saddle point \\
\hline
\end{tabular}

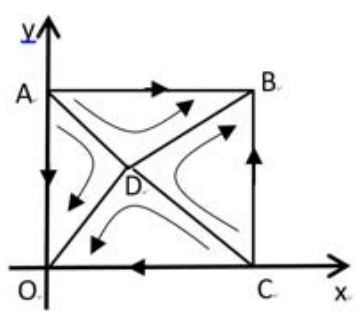

(1) $0<x^{*}<1,0<y^{*}<1$

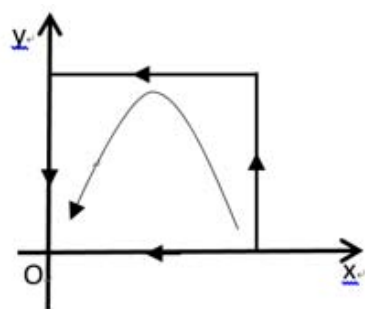

(2) $0<x^{*}<1, \underline{L}^{*}>1$

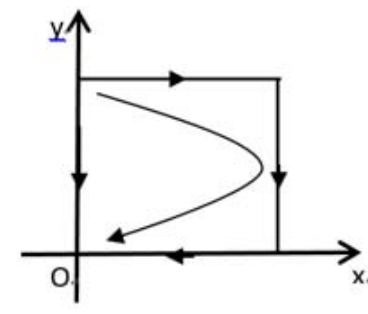

$(3) x^{*}>1,0<y^{*}<1$

Figure3: System dynamic evolution figures of three different situations

\section{Evolutionary game stability simulations}

We use the Matlab to simulate the evolutionary game. So when the parameters change, we can analyze the evolutionary trend of the patient system and the doctor system visually. Now we will analyze the evolutionary trend of cost, risk and public praise of the knowledge sharing.

\section{Volume 5 Issue 4, April 2016}




\section{International Journal of Science and Research (IJSR) \\ ISSN (Online): 2319-7064}

Index Copernicus Value (2013): 6.14 | Impact Factor (2015): 6.391

First, set the initial values of model parameters:

$\mathrm{T} 1=0.5 ; \mathrm{U} 1=0.7 ; \mathrm{K} 2=80 ; \mathrm{P}=50 ; \mathrm{K} 1=100 ; \mathrm{F}=0.5 ; \mathrm{C} 1=30 ; \mathrm{E}=3 ; \mathrm{T} 2$

$=0.5 ; \mathrm{U} 2=0.9 ; \mathrm{n}=1 ; \mathrm{D}=3 ; \mathrm{C} 2=20 ; \mathrm{x}^{*}=0.59, \mathrm{y}^{*}=0.5$.

The evolutionary result in setting different initial knowledge sharing proportions is shown as figure 4 .

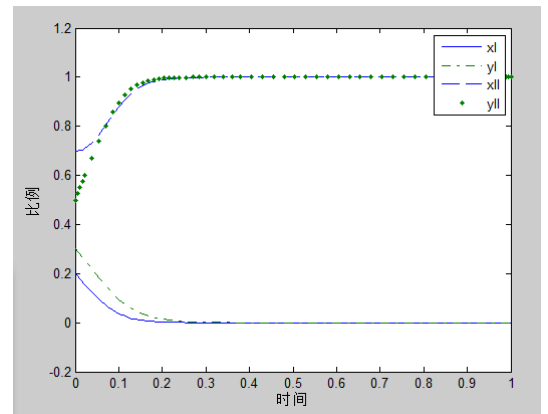

Figure4:different initial proportion

Set both sides' initial proportions to be $(0.2,0.3)$ and ( 0.5 , 0.7 ). The experiment result is shown in figuer 4 . From the figuer we can find the track of the system and the state's change from convergent to balanced is relation to the initial state. Such as the point $(0.2,0.3)$ isn't in ABCD, evolutionary equilibrium converge to point $\mathrm{O}(0,0)$. When the point $(0.5,0.7)$ is in $\mathrm{ABCD}$, evolutionary equilibrium converge to point $\mathrm{B}(1,1)$. In the evolutionary process, we can see from the figuer that at first it rises slowly, and then goes fast, rate of convergence is fast when it gets closer to the balanced state. When other parameters don't change, doctors' public praise is as a result of the evolutionary is shown as figure 5 .

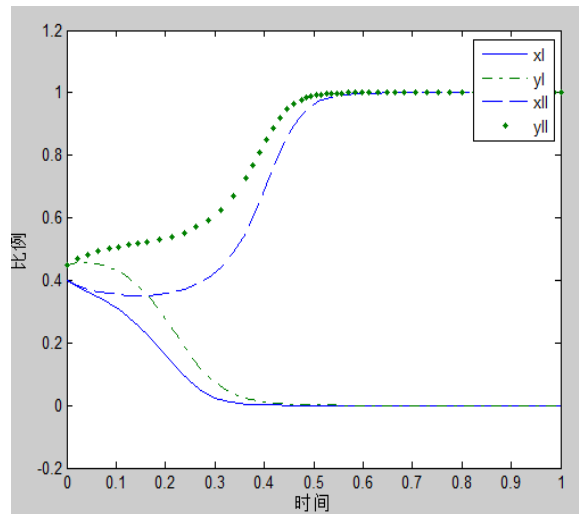

Figure 5: public praise revenue

The doctor's public praise changes from $\mathrm{D}=3$ to $\mathrm{D}=6$. We can see from the figuer 5 , along with public praise increase to $\mathrm{B}(1,1)$, if the doctor doesn't want to share his knowledge actively, according to the parameters we have set his loss will be double. The more the doctor is willing to pay to keep his public praise, the higher knowledge sharing positivity is. The impact simulation diagram of the revolutionary result is shown as figure 6 when other parameters remain unchanged but patients' knowledge sharing risks change.

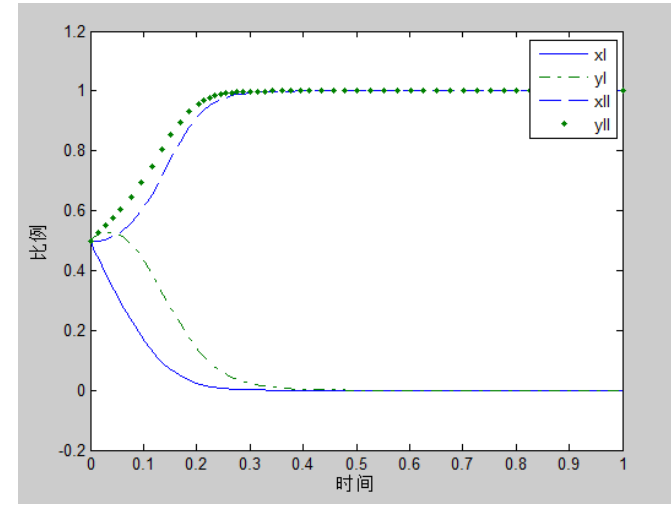

Figure 6: risk

The loss of the patient's risks has influences on evolutionary results. We can see from the figuer 6 , along with the decease of risk loss, patients will be more willing to sharing. in the knowledge sharing process, patients 's privacy and self-information are probably put in the air .it may result in unpredictable lost not only in economic but also in their daily lives.so in knowledge sharing patients take care more about the privacy betrayal risk than the money they paid.

\section{Management Suggestions}

We can find the influence factor that influent knowledge sharing between doctors and patients from the evolutionary game model above.

(1)First, the online medical treatment needs to enhance the propaganda. The initial proportion of online consulting function is increased. The beginning state is in the quadrangle $\mathrm{ADCB}$, the system evolutionary offers the convenient condition to knowledge sharing to users. At present, haodf has already offered phone-consult. In the future, the picture and video consult function can be finished. So we can not only decline the sharing cost but also improve the diagnosis accuracy [14]. What's more, the service providers need to build friendly relationship and gain the trust. Adding a function in the online medical community will change the weak relationship to the strong one. The great trust in users, the problems and conflicts in the process of knowledge sharing can be resulted effectively, the credibility is increased and the cost of knowledge sharing is decreased accordingly.

(2) The web must enhance keeping the privacy of the patients. Searching the medical web we will find that the security isn't enough. However, the risk is the main factor which patients pay attention to. So decrease the knowledge sharing risks and increase its probability.

(3)Before consulting, patients can improve their cognitive abilities about the process of their disease. When they choose doctors, they can improve the understanding and the trust to the doctors by searching doctors' data and classic disease cases, consulting ward-mates. It will push patients to share knowledge actively. 


\section{International Journal of Science and Research (IJSR) \\ ISSN (Online): 2319-7064 \\ Index Copernicus Value (2013): 6.14 | Impact Factor (2015): 6.391}

(4)The online medical web is able to apply consultation to patients [15]. Because of the limit of technology, in this moment it is impossible to complete the online service from consulting to get medicines totally. Convenient is the best advantage of the online medical service. Doctors can know and analyze the condition conveniently and continually. So management concept of the online medical treatment must pay attention to patients and curative effect.

\section{References}

[1] Faraj S.,Johnson S. L. (2011). Network Exchange Patterns in Online Communities [J]. Organization Science, 22 (6): 1464-1480.

[2] FarajS., Jarvenpaa S. L. (2011).Majchrzak A. Knowledge Collaboration in Online Communities [J]. Organization Science, 22 (5): 1224-1239.

[3] ZhuR., DholakiaU. M., Chen X., et al.(2012).Does Online Community Participation Foster Risky Financial Behavior?. Journal of Marketing Research, 49 (3): 394-407.

[4] Bateman P. J., Gray P. H.,Butler B. S. (2011).Research Note-The Impact of Community Commitment on Participation in Online Communities . Information Systems Research, 22 (4): 841-854.

[5] Faraj S.,Johnson S. L. (2011). Network Exchange Patterns in Online Communities. Organization Science, 22 (6): 1464-1480.

[6] Katona Z., Zubcsek P. P.,Sarvary M. (2011).Network Effects and Personal Influences: The Diffusion of an Online Social Network. Journal of Marketing Research, 48 (3): 425-443.

[7] Liu Xiaoxiao (2014). The impact of online doctor repution and doctor effort on consultation amount. Ms D Thesis. Harbin:Harbin Institue of Technology,

[8] Yang L., Tan Y. (2010). Feeling Blue So Going Online: An Empirical Study on Effectiveness of Virtual Social Networking . proceedings of the Workshop on Health IT and Economics, F.

[9] Greene J. A., Choudhry N. K., Kilabuk E., et al. (2011).Online social networking by patients with diabetes: a qualitative evaluation of communication with Facebook. Journal of general internal medicine, 26 (3): 287-292.

[10] Sillence E. (2013).Giving and receiving peer advice in an online breast cancer support group. Cyberpsychology, Behavior, and Social Networking, 16 (6): 480-485.

[11]Cohen R., Elhadad M.,Birk O. (2013). Analysis of Free Online Physician Advice Services. PloS one , 8 (3): e59963.

[12]Zeng Jianghong,Cui Xiaoyun.(2015). Study on Parent_subsidiary Corporation Governance of Enterprise Groups Based on Evolutionary Game.Management Science in China,(02):148-149.

[13]Chen Zhisong,Wang Huimin,Qiu Lei,Chen Junfei.(20008).Evolutionary Game Analysis of Water Resources Allocation in river basin.Management Science in China,(06):176-183.
[14] Faraj S., Jarvenpaa S. L.,Majchrzak A. (2011) Knowledge Collaboration in Online Communities. Organization Science, 22 (5): 1224-1239.

[15] LIN M J, HUNG S W, CHEN C J. (2009). Fostering the determinants of knowledge sharing in professional virtual communities. Computers in Human Behavior,25 (4) : 929-939. 